\title{
Remote Sensing Data Feature Analysis Using Spatial Linear Embedding (SLE)
}

\author{
Lifang Xue ${ }^{a}$,Xiushuang $\mathrm{Yi}^{\mathrm{b}}$,Xiumei Liưcand Jie $\mathrm{Li}^{\mathrm{d}}$ \\ Computer Center, Northeastern University, Shenyang, China

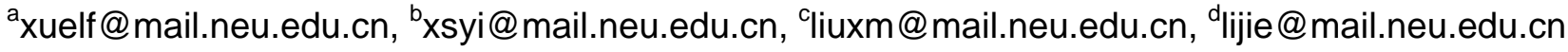

Keywords:Spatial linear embedding; Remote sensing data feature analysis; Manifold learning.

\begin{abstract}
Dimensionality reduction has been used to reduce the complexity of the representation of remote sensing data. In this paper, a novel remote sensing data feature analysis method is proposed based on an improved manifold learning algorithm--spatial linear embedding. The purpose of feature extraction is to reduce the dimensionality of the remote sensing data while preserving the significant information. Compared with LLE, spatial linear embedding method emphasizes the relation of neighboring pixels spatially to increase system efficiency. The method makes up the shortage that LLE ignores the relation of neighboring pixels spatially which is extremely important for remote sensing data. In this paper we have obtain experiment results from the analysis of remote sensing data using PCA and spatial linear embedding. The results show that the SLEcan give significantly higher accuracies than the linear method of PCA.
\end{abstract}

\section{Introduction}

Remote sensing data are characterized by the high spectral resolution that offers many wavelength channels per pixel[1]. These continuous spectral channels increase the efficiencies for feature extracting, classifying and clustering over an area. In remote sensing data, nonlinear properties are intrinsic. The nonlinear characteristics of remote sensing data embody nonlinear topological structures in feature space and this feature may disappear if input data are projected into a space described by eigenvectors. The nonlinear structures are important for information extraction from remote sensing data.A low dimensional submanifold may have a significant nonlinear structure that linear methods couldn't discover.

Dimensionality reduction methods have been used to dissolve the problems of serious spectral correlation and the 'Hughes phenomenon' for classification of remote sensing data. Nonlinear manifold approaches presume that the original high dimensional input data virtually may be situated on a low dimensional manifold, which is defined by local geometric differences between data samples. Local manifold learning methods including, locally linear embedding (LLE) [2], preserve local geometry isometric feature. The advantage of these local manifold learning methods is that they have computational efficiency because of only requiring sparse matrix computations. A number of exploratory studies $[3,4,5,6]$ have shown that manifold learning algorithms can be used to analysis and classify remote sensing data successfully in a low dimensionality space.

In this paper, we apply one of these algorithms-Spatial linear embedding -to the feature analysis and classification of remote sensing data. Spatial linear embedding method makes use of both spectral and spatial information implicated in remote sensing data to increase system efficiency. The method makes up the shortage that LLE ignores the relation of neighboring pixels spatially which is extremely important for remote sensing data. This important spatial information is recognized as characteristics of geographical data, including the relation of neighboring samples to the target data sample. There has few feature analysis approaches to exploit spatial information because remote sensing data have high dimensionality and the spectral and spatial heterogeneity [7]. The ideal of spatial linear embedding is to group the neighboring pixels into spectrally homogeneous objects. The ability of the algorithm to discover low dimensional structure within remote sensing data and classification results are evaluated and compared. 


\section{Remote Sensing Data Feature Extraction by Spatial Linear Embedding}

\section{Locally Linear Embedding (LLE).}

The LLE proposed by Sam T.Roweis [2] is an effective algorithm for the dimensionality reduction of high dimensionality data. Given high dimensionality data inputs $\left\{\vec{X}_{i}\right\}_{i=1}^{N}$ in $D$ dimensions, LLE algorithm tries to discover low dimensionality data outputs $\left\{\vec{Y}_{i}\right\}_{i=1}^{N}$ in the condition of $d \square D$.

The algorithm has three steps as followed:

Firstly, the algorithm attempts to compute neighbors for every high dimensionality input $\vec{X}_{i}$. Neighbors may be computed according to the simplest $K$ nearest neighbors based on Euclidean distance.

Secondly, the algorithm appeals to the thought that neighboring input samples situate on a locally linear patch of the manifold. For every input data $\vec{X}_{i}$, LLE compute series of linear coefficients to reconstruct the input data from neighboring samples. The total reconstruction error of the inputs is measured by the cost function:

$$
\varepsilon(W)=\sum_{i}\left|\vec{X}_{i}-\sum_{j} W_{i j} \vec{X}_{j}\right|^{2}
$$

The weight $W_{i j}$ saves the contribution of $\vec{X}_{j}$ to the linear reconstruction of $\vec{X}_{i}$.

Thirdly, the algorithm tries to compute a low dimensionality embedding that is characterized by the same reconstruction weights. In general, we choose the low dimensionality outputs $\vec{Y}_{i}$ to minimize the embedding cost function:

$$
\Phi(Y)=\sum_{i}\left|\vec{Y}_{i}-\sum_{j} W_{i j} \vec{Y}_{j}\right|^{2}
$$

We can compute the bottom $d+1$ eigenvectors of the $N \times N$ matrix $(I-W)^{T}(I-W)$ to obtain The minimum of Eq.(2). The bottom eigenvector is discarded, and the remainder $d$ eigenvectors minimizes Eq.(2) by the constraints of centering and orthogonality.

\section{Spatial Linear Embedding(SLE).}

Spatial linear embedding makes use of both spectral and spatial information implicated in remote sensing data to increase system efficiency. So except for choosing neighbors on the basis of Euclidean distance in remote sensing data, the predecessors and successors of an input sample can be utilized to determine reconstruction weights. We can define the neighborhood of an input sample by both spectral and spatial terms, and choose the $K$ closest points along the spectral and spatial coordinate system.

Error equations of spatial linear embedding for reconstruction and embedding can be expressed and optimized according to LLE.

$$
\begin{aligned}
& \xi(X, W)=\operatorname{trace}\left(\left(I^{(N)}-W\right)^{T}\left(I^{(N)}-W\right) X^{T} X\right) \\
& \operatorname{trace}\left(M Y^{T} Y\right)
\end{aligned}
$$

\section{Experiment and Result Analysis}

To investigate SLE's potential on extracting features from remote sensing data, we used the image data called SubCanb.BSQ. The image segment is 100 lines of 400 pixels each and contains 6 Thematic Mapper bands (Bands 1, 2, 3, 4, 5, \& 7).

Because of the memory constraint, we were not able to process the entire image at once. A $100 \times 100$ pixel subset of the image was selected in this study. The entire image and the subset are shown in Fig.1. The size of the input data is $n \times d$, for $n=10000$ and $d=6$, representing the number 
of data points and the input data dimensionality, respectively. The number of nearest neighbours for reconstruction was experimentally set to 13 . That is: $k=13$. The output dimensionality was set to 5 . That is: $r=5$.

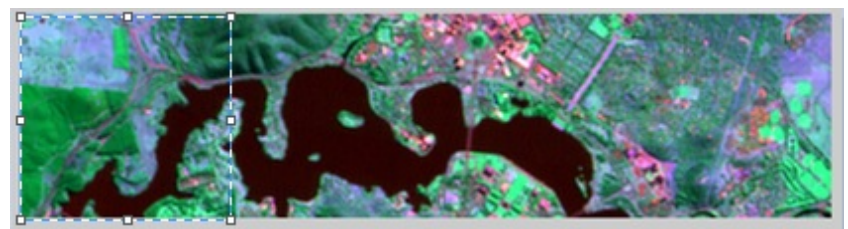

Figure1. SubCanb image (the selected subset is enclosed by the dotted square)

\section{Information content preservation.}

The first experiment conducted was on information content preservation. The information content represented by the first PCA-band image is calculated as the eigenvalue corresponding to the first eigenvector of the data covariance matrix. So are the otherPCA-band images. Because the first few eigenvectors account for the majority of the total variance, the corresponding PCA-band images maintain the major part of the information content in the image. The first three PCA-band images are shown in Fig.2.
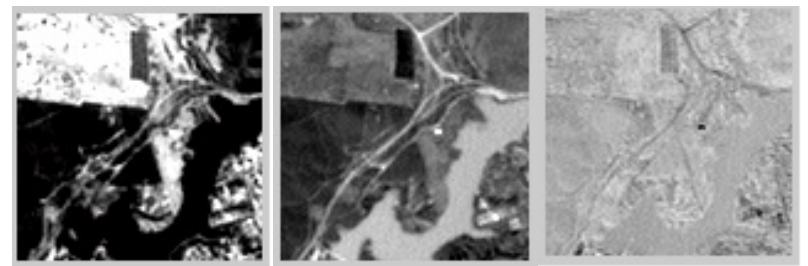

Figure2. PCA-band images for the subset of SubCanb image (left=PCA 1; middle=PCA2;right=PCA3)

To investigate SLE's ability on information preservation, we compared SLE-band images with PCA-band images of the same SubCanb data show in Fig.1. The corresponding SLE results are given in Fig.3.

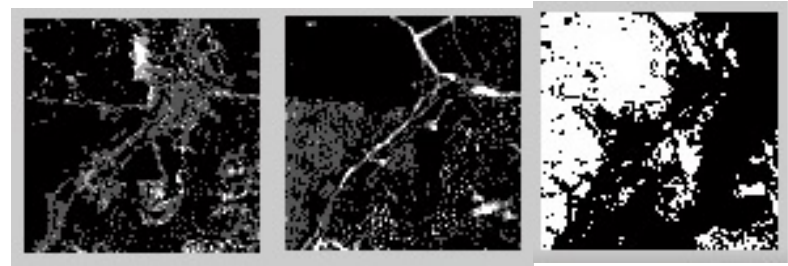

Figure3. SLE-band images for the subset of SubCanb image (left= SLE 1; middle= SLE 2;right= SLE 3)

This comparison revealed that: the three SLE-band images preserved similar amounts of spatial information as that preserved by the PCA-band images, which are believed to represent the major information content of the original remote sensing image.

Pure pixel identification.

The experiment pursued in this study was to test if we would exploit SLE's ability on preserving local topological structure of each data point to facilitate pure pixel identification. Statistically, pure pixels often occur at corners or tips of the data cloud expressed in a scatter plot. Feature extraction is often employed to reduce data dimensionality, and make it manageable when expressed in a scatter plot. Fig. 4 is a 3D-scatter plot constructed with the first 3 PCA-band images. However, this featureless data cloud makes it impossible for us to spot more than one pure pixel. In contrast, Fig.5 is another 3D-scatter plot built with the first 3 SLE band-images. By tracking down the pixels located at the four tips of the data cloud, pure pixels are readily identified. It turned out that these were four endmembers - water, soil, plant or concrete. 

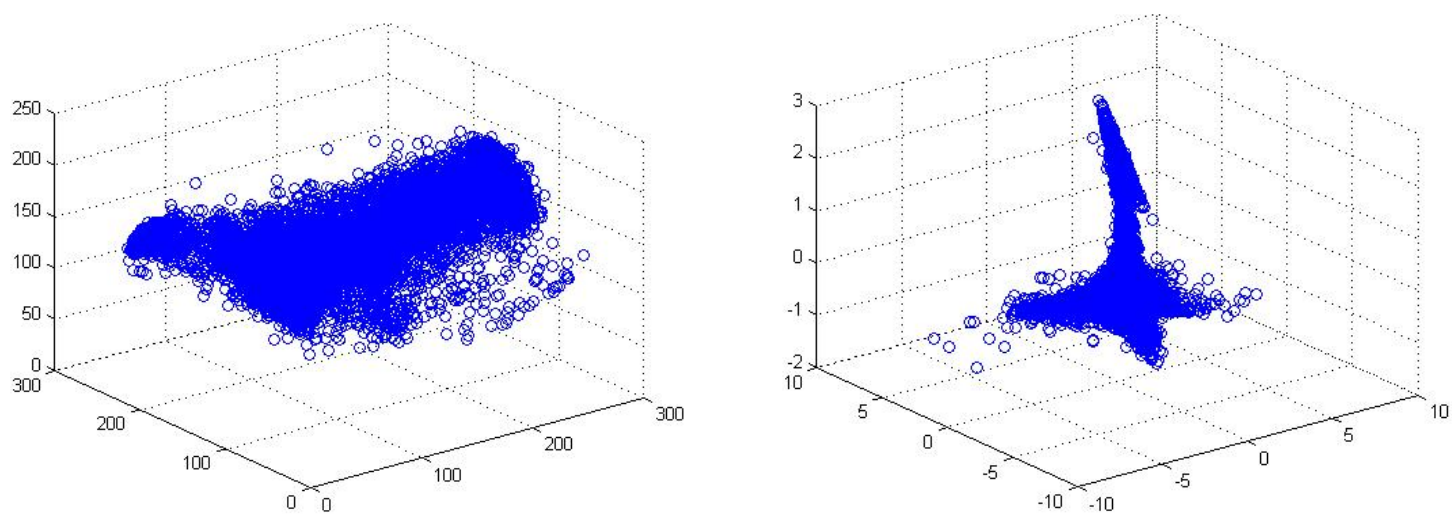

Figure4.3D-scatter plot with PCA 1, 2 and 3Figure5.3D-scatter plot with SLE 1, 2 and 3

\section{Conclusions and Future Work}

In this paper, we have presented results from the remote sensing data analysis using spatial linear embedding. We observed that the nonlinear embeddings of spatial linear embedding is promising for remote sensing data feature extraction.Future considerations will be given to thoroughly examining SLE for hyperspectral data information extraction and optimizing SLE to process full scene hyperspectral images.

\section{Acknowledgments}

This research has been partially supported by the Fundamental Research Funds for the Central Universities N110216001, N100316001 and NNSF61502092.

\section{References}

[1] M. A. Karaska, R. L. Huguenin, J. L. Beacham, M. Wang, J. R. Jensen, and R. S. Kaufmann, "AVIRIS Measurements of Chlorophyll, Suspended Minerals, Dissolved Origanic Carbon, and Turbidity in the Neuse River, North Carolina,” Photogrammetric Engineering \& Remote Sensing, Vol.70, No.1, January 2004, pp. 125-133.

[2] S.T. Roweis and L.K. Saul, "Nonlinear dimensionality reduction by locally linear embedding," Science, 2000, vol. 290(5500), pp. 2323-2326.

[3] T. Han, D. G. Goodenough, "Nonlinear Feature Extraction of Hyperspectral Data Based on Locally Linear Embedding (LLE)," Geoscience and Remote Sensing Symposium, Vol.2, pp. 1237-1240, 2005.

[4] Viren Jain and Lawrence K. Saul, "Exploratory analysis and visualization of speech and music by locally linear embedding,” ICASSP, 2004, vol. 3, pp. 984-987.

[5] D. Lunga, S. Prasad, M. M. Crawford and O. Ersoy, "Manifold-Learning-Based Feature Extraction for Classification of Hyperspectral Data," IEEE Signal Processing Magzine, 2014, January, pp.55-66.

[6] L. Ding, P. Tang and H.Y. Li, "Subspace Feature Analysis of Local Manifold Learning for Hyperspectral Remote Sensing Images Classification,” Applied Mathematics \& Information Sciences, 2014, vol. 8, No.4, pp.1987-1995.

[7] X. Huang, L. Zhang, "A Comparative Study of Spatial Approaches for Urban Napping using hyperspectral ROSIS images over PVI City, northern of Italy," International Journal of Remote Sensing, 2009, vol. 30, No.12, pp.3205-3221. 